\title{
Inhibition of Amyloidogenesis
}

National Cancer Institute

\section{Source}

National Cancer Institute. Inhibition of Amyloidogenesis. NCI Thesaurus. Code C45553.

Inhibition of Amyloidogenesis is a process that prevents the formation or growth of amyloid structures. 